\title{
COnE).(ÖSS
}

CIÊNCIA E TECNOLOGIA

\section{ACESSIBILIDADE DE INTERFACES WEB PARA CUIDADORES COM BAIXA ESCOLARIDADE: ASPECTOS E REFLEXÕES}

\author{
Eliane Pinheiro Capra, Simone Bacellar leal Ferreira, João Marcelo Marques, \\ SAMUEL SANTOS PORTELA
}

Universidade Federal do Estado do Rio de Janeiro - UNIRIO <aelianecapra@yahoo.com.br>,<simone@uniriotec.br>,<joao.marques@uniriotec.br>

$<$ samuelsportela@gmail.com>

DOI: 10.21439/conexoes.v15i0.2087

\begin{abstract}
Resumo. A evolução da internet trouxe novas perspectivas de interação e comunicação entre as pessoas, bem como muitos desafios. A interação com conteúdo textual pode ser impactada, especialmente para os analfabetos funcionais, que são pessoas com comprometimento nas habilidades de leitura e escrita ou com baixa escolaridade, de acordo com critérios estabelecidos pela UNESCO. Dentre esses usuários, pode-se encontrar os cuidadores de idosos, uma vez que boa parcela é considerada analfabeta funcional, por causa da baixa escolaridade. Este artigo trata de um levantamento de caráter exploratório, com objetivo de analisar as contribuições disponíveis na literatura acerca da acessibilidade web para esse público, identificando fatores que podem tornar a web acessível aos cuidadores analfabetos funcionais e possíveis lacunas para pesquisas futuras. Para isso, foi realizado um mapeamento sistemático seguindo a abordagem Systematic Literature Review (SLR), a fim de analisar artigos publicados entre 2000 e 2019 em quatro bases acadêmicas internacionais. Foram extraídos 58 artigos e, após triagem e leitura de 23 trabalhos, foram identificadas 12 pesquisas relacionadas ao tema. O mapeamento possibilitou a comparação entre as publicações e a identificação de aspectos que podem influenciar no desenvolvimento de interfaces acessíveis a cuidadores analfabetos funcionais, além de revelar importantes contribuições sobre recomendações, modelos, sistemas e aplicações. Espera-se que os resultados desta pesquisa possam contribuir para a conscientização dos pesquisadores sobre a importância do tema na comunidade de Interação Humano Computador (IHC).
\end{abstract}

Palavras-chaves: Acessibilidade Web. Analfabetismo Funcional. Cuidadores de Idosos. Inclusão.

\section{WEB ACCESSIBILITY INTERFACES FOR LOW LITERACE ELDERLY CAREGIVERS: ASPECTS AND REFLECTIONS}

\begin{abstract}
The evolution of internet has implied new interaction and communication perspectives among people as well as new challenges. The interaction with text content may be impacted, especially for functional illiterates, who are people with impairment in reading and writing skills or with low schooling, according to the criteria set by UNESCO. Among these users, we can find elderly caregivers, once a great part of them is considered functional illiterate, due to low schooling. This article addresses an exploratory search, with the purpose of analyzing the available contributions in literature about web accessibility for this public, identifying elements that may make web accessible for functional illiterate caregivers and possible blanks for future research. To this end, it was accomplished a systematic mapping, by following the approach Systematic Literature Review (SLR), in order to analyze published articles between 2000 and 2019 in four international academic bases. Thus, 58 articles were extracted and, after screening and reading of 23 works, were identified 12 researches related to the subject. The mapping made possible the comparison among publications and the identification of elements that may influence the development of accessible interfaces for functional illiterate caregivers, besides important contributions about recommendations, models, systems, and applications. It is expected that the results from this research can contribute to the researchers' awareness about the importance of the subject in Human-Computer Interaction (HCI) Community.
\end{abstract}

Keywords: Web Accessibility. Functional Illiteracy. Elderly Caregivers. Inclusion. 


\section{INTRODUÇÃO}

A evolução das tecnologias de comunicação, em especial a internet, trouxe novas perspectivas de comunicação entre as pessoas. Ela ampliou potencialmente a capacidade de interação, mudando a forma como os indivíduos vivem e se relacionam. No entanto, mesmo trazendo inúmeras facilidades e benefícios para comunicação humana, o mundo conectado pela web traz uma série de desafios (SILVA et al. 2018).

Um dos desafios é o uso da internet por pessoas com diferentes dificuldades, como a população com comprometimento nas habilidades de leitura e escrita, chamada de analfabeta funcional (VÁGVÖLGYI et al. 2016; BARBOZA; NUNES, 2007; RIBEIRO, 1997). A disseminação de conteúdo puramente textual nos sítios da web pode comprometer a compreensão das informações, dificultando o entendimento dos documentos para as pessoas com essas dificuldades (PRAKASH; ANANTHAN; RAJAVARMAN, 2014).

No Brasil, seguindo a recomendação da United $\mathrm{Na}$ tions Educational, Scientific and Cultural Organization (UNESCO), são classificadas como analfabetas funcionais as pessoas com menos de quatro anos completos de escolaridade (IBGE, 2010, INEP, 2020, RIBEIRO; VÓVIO; MOURA, 2002). Considerando essa classificação, estima-se que $16,6 \%$ da população brasileira é analfabeta funcional (INEP, 2020). Especialmente no campo da saúde, considerando a escolaridade, boa parcela dos cuidadores de idosos são analfabetos funcionais (JESUS; ORLANDI; ZAZZETTA, 2018), o que pode comprometer algumas tarefas relacionadas ao trato de idosos (COLOMÉ et al., 2011, ALMEIDA 2017; JESUS; ORLANDI; ZAZZETTA, 2018).

Embora as estatísticas não apresentem números favoráveis, os analfabetos funcionais em geral destacamse por realizar inúmeras atividades na web (CETIC-C6 2019). Ou seja, esses usuários podem ser tratados como potenciais consumidores de serviços e produtos na web (CAPRA; FERREIRA; TORRES, 2020).

Diante da crescente difusão da informação em meios digitais, estudos foram realizados sobre a acessibilidade $w e b$, com o objetivo de melhorar a qualidade de acesso de pessoas com diferentes tipos de limitações (GRANATTO; PALLARO; BIM, 2016). Entretanto, o analfabetismo funcional ainda é um tema pouco explorado em pesquisas acadêmicas (GRANATTO; PALLARO; BIM, 2016).

Visto que o analfabetismo funcional é um tópico social importante a ser considerado no desenvolvimento de uma interface e que boa parcela dos cuidadores de idosos tem baixa escolaridade, este artigo tem como objetivo analisar as contribuições disponíveis na literatura da área da Interação Humano Computador (IHC) para esse público, a fim de identificar os aspectos que podem tornar a web acessível aos cuidadores analfabetos funcionais e também possíveis lacunas para pesquisas futuras. Para isso, os autores realizaram um mapeamento sistemático seguindo a abordagem Systematic Literature Review (SLR) (KITCHENHAM, 2004, KITCHENHAM; CHARTERS, 2007). Foram analisados artigos completos publicados em quatro bases acadêmicas internacionais entre os anos 2000 e 2019.

\section{REFERENCIAL TEÓRICO}

No contexto da web, a acessibilidade se refere à possibilidade de prover acesso e oportunidades iguais a qualquer grupo de usuários (FERREIRA; NUNES. 2008, NIELSEN, 2007; ELLCESSOR, 2015).

Dentre os grupos de pessoas beneficiadas pela acessibilidade web estão: os idosos, que possuem limitações naturais acarretadas pelo tempo (SACRAMENTO et al., 2019); cegos, surdos ou com alguma deficiência cognitiva (WAI 2020); pessoas com dificuldades relacionadas à habilidade de leitura e escrita, como os analfabetos funcionais (CAPRA; FERREIRA; TORRES 2020).

A acessibilidade web caracteriza-se pela possibilidade de diferentes pessoas, independentemente de suas capacidades físico-motoras, perceptivas, culturais e sociais, poderem utilizar a internet (CAPRA; FERREIRA; TORRES, 2020, FERREIRA; NUNES, 2008, WAI, 2020), incluindo os sistemas de informação, sites, chats, correios eletrônicos, entre outros (FERREIRA; NUNES, 2008).

\subsection{Conceitos sobre Analfabetismo Funcional}

O "analfabetismo" foi o termo empregado para designar a condição daqueles que não sabiam ler nem escrever (RIBEIRO, 1997). O uso do termo "analfabetismo funcional" em alguns países deveu-se basicamente à UNESCO no fim da década de 1970, com objetivo de padronizar as estatísticas de ensino e influenciar políticas educativas (RIBEIRO, 1997).

A fim de estabelecer quais seriam as demandas referentes à alfabetização em relação às diferentes realidades de cada país, a UNESCO sugeriu que se tomasse como indicador de analfabetismo funcional o número de escolarização do indivíduo, que foi seguido por alguns países (RIBEIRO, 1997). O Canadá considera nove anos de escolaridade, enquanto Espanha considera seis anos de escolaridade (RIBEIRO, 1997).

Seguindo a recomendação da UNESCO, o Brasil começou, por meio das pesquisas censitárias realiza- 
das pelo Instituto Brasileiro de Geografia e Estatística (IBGE), a classificar como analfabetas funcionais as pessoas com menos de quatro anos completos de escolaridade (IBGE, 2010, RIBEIRO; VÓVIO; MOURA 2002). O IBGE continua investigando indicadores sobre escolaridade, dentre eles, pode-se citar: o analfabetismo de pessoas com mais de 15 anos, nível de instrução e número médio de anos de estudos de adultos e a taxa de escolaridade por faixas etárias (PNAD, 2017). O Instituto Nacional de Estudos e Pesquisas Educacionais Anísio Teixeira (INEP) também utiliza o termo analfabetismo funcional e segue a classificação de escolaridade em suas pesquisas (INEP, 2020).

\subsection{Cenário Brasileiro sobre Analfabetismo Funci- onal e Cuidadores de Idosos}

O INEP divulga, a cada dois anos, o Relatório de Monitoramento das Metas do Plano Nacional de Educação (PNE), cujo objetivo é publicar os estudos para aferição do cumprimento das vinte metas de educação estabelecidas para o cenário brasileiro (INEP, 2020). As metas são aferidas de acordo com diferentes fontes públicas, dentre elas, os dados estatísticos de educação publicados pelo IBGE. As regiões Norte e Nordeste estão acima da média brasileira, que é de 16,6\%. O índice mais baixo de analfabetismo funcional é da Região Sudeste, com 11,5\% (INEP, 2020).

O Instituto Paulo Montenegro, instituição vinculada ao IBOPE, e a Ação Educativa, uma organização nãogovernamental, vêm realizando, desde 2001, pesquisas que medem os níveis de alfabetismo da população de 15 a 64 anos em diferentes séries escolares (INAF 2018). Tais pesquisas fundamentam o Indicador de Alfabetismo Funcional (INAF), criado em 2009, e consistem em testes cognitivos e questionários de contextualização sociodemográfica, econômica, cultural e educacional por meio de entrevistas domiciliares (INAF 2018). A amostra dos participantes dos testes é estratificada proporcionalmente à população brasileira.

Segundo o INAF, o analfabetismo funcional em 2001 atingia 39\% da população brasileira, incluindo pessoas com ensino médio ou superior. O último relatório do INAF, publicado em agosto de 2018, o índice de analfabetismo funcional encontra-se em $29 \%$ (INAF, 2018). Conforme Figura 1. a queda é positiva, porém o percentual ficou inalterado de 2009 a 2015, voltando a crescer em 2018.

Embora estejam presentes na literatura iniciativas no desenvolvimento de interfaces acessíveis para esse público, este ainda enfrenta desafios em decorrência das dificuldades na leitura e escrita, como a busca por emprego (DIEL, 2014; KHAN et al., 2017), especialmente
Figura 1: Evolução do Indicador de Alfabetismo Funcional.

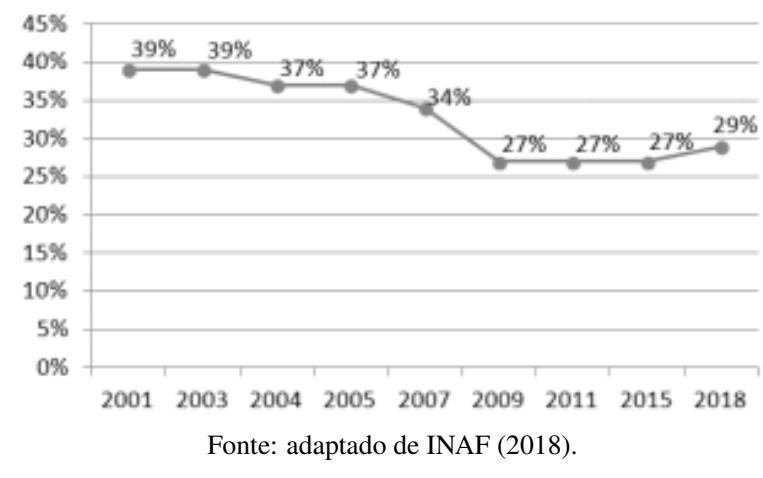

no trato de cuidados da saúde do idoso (COLOMÉ et al., 2011;, ALMEIDA, 2017; JESUS; ORLANDI; ZAZZETTA, 2018).

Nos últimos anos, o Brasil tem passado por mudanças demográficas influenciadas por vários fatores, dentre eles, o aumento da expectativa de vida (CARDOSO et al. 2018), com uma estimativa de 73,5 milhões de idosos em 2060 (FURTADO et al. 2020). O processo de envelhecimento traz mudanças na qualidade de vida do cidadão, impactando a independência nas atividades diárias e demandando suporte da família para o cuidado do idoso (COLOMÉ et al., 2011, CARDOSO et al. 2018). Nesse contexto, o cuidador ganha destaque, pois ele desempenha a função necessária para o cuidado do idoso, proporcionando-lhe bem-estar, segurança, conforto e incentivando autonomia e independência (CARDOSO et al., 2018). Além disso, em pesquisa recente, $24 \%$ dos idosos informaram que em caso de incapacidade, prefeririam ser cuidados por pessoas de fora da família (FURTADO et al. 2020).

A maior parte dos cuidadores são conhecidos ou da própria família, e a escolaridade está predominantemente entre um e quatro anos de estudos $(85,9 \%)$ e 4,4\% são analfabetos (JESUS; ORLANDI; ZAZZETTA, 2018). A baixa escolaridade dos cuidadores pode deixar os idosos vulneráveis às tarefas de cuidado, tais como: uso de serviços de emergência (ALMEIDA. 2017); auxílio na ministração de medicamentos (COLOMÉ et al. 2011); acompanhamento de consultas; recebimento e repasse de orientações médicas (COLOMÉ et al., 2011; ALMEIDA, 2017; JESUS; ORLANDI; ZAZZETTA, 2018). Essas tarefas, se não forem entendidas corretamente, podem levar ao comprometimento da saúde do idoso (COLOMÉ et al. 2011, ALMEIDA. 2017). 


\subsection{Acessibilidade web para Analfabetos Funcio- nais}

No contexto brasileiro da web, mesmo com as dificuldades de leitura e escrita, boa parcela desse público acessa a internet para realizar diversas atividades, inclusive para buscar informações sobre produtos, serviços e saúde (CETIC-C6, 2019). Entretanto, o desempenho desses usuários na navegação de sites de busca e recuperação de informações é inferior aos usuários com nível de escolaridade mais alto (KODAGODA; WONG 2008). Este fato pode impactar os cuidadores de idosos, uma vez que tendem a pesquisar informações sobre trato do idoso (ALMEIDA, 2017).

Pesquisadores e especialistas buscam estudar formas de facilitar o acesso à web por esse público, desenvolvendo tecnologias e recursos não textuais para auxiliar a navegação deles nas páginas de internet. As tecnologias assistivas são programas ou dispositivos voltados a facilitar o acesso à web, proporcionando-lhes habilidades funcionais ou ampliando essas habilidades (FERREIRA; NUNES, 2008). Outros recursos auxiliam desenvolvedores na produção de textos mais simples, a fim de facilitar a leitura do conteúdo textual da web (MARTINS et al., 2003; HUENERFAUTH, 2002, BARBOZA; NUNES 2007). Ressaltam-se, ainda, as pesquisas relacionadas à avaliação da acessibilidade web com a participação de analfabetos funcionais, uma vez que é importante avaliar a acessibilidade de um site com a participação de usuários (CAPRA et al., 2012b).

No fim da década de 90 , foram iniciadas as primeiras pesquisas sobre a acessibilidade $w e b$ no âmbito nacional e internacional. Em 1999, com o objetivo de tornar o conteúdo web acessível para todos os tipos de usuários, o W3C (World Wide web Consortium) criou o WAI (web Accessibility Initiative), responsável pelo desenvolvimento de padrões para tornar a web acessível para todos, além de elaborar materiais que auxiliam a entender e implementar a acessibilidade (WAI, 2020). O W3C/WAI desenvolveu o WCAG (web Content Accessibility Guidelines), um documento com catorze recomendações para acessibilidade para conteúdo web, o qual se encontra na versão 2.1 atualmente (WCAG 2018). O documento é estruturado em quatro princípios: perceptível, operável, compreensível e robusto (WCAG, 2018). O princípio “compreensível” pode ser considerado o que mais possui recomendações relacionadas ao público analfabeto funcional, visto que uma de suas diretrizes é tornar o conteúdo textual da web legível e compreensível (WCAG, 2018).

Mesmo com iniciativas para tornar a $w e b$ acessível, os analfabetos funcionais ainda encontram barreiras no acesso à internet. Por exemplo, em navegação em sites de busca, esses usuários levam oito vezes mais tempo para realizar uma tarefa do que usuários alfabetizados e podem ficar treze vezes mais propensos a se perderem na realização da uma tarefa de busca (KODAGODA; WONG , 2008). Além disso, eles levam, em média, um terço a mais na navegação de uma página do que usuários alfabetizados e empregam uma estratégia menos focada, visitando oito páginas a mais para obter as informações necessárias; e utilizam o botão voltar do navegador treze vezes a mais do que os usuários alfabetizados, o que eleva o número de "revisitas" a uma mesma página (KODAGODA; WONG, 2008).

Ainda sobre as dificuldades de acesso à web por esse público, em 2013 (BENJAMIN et al., 2013) pesquisadores realizaram testes simples de verificação textual em serviços de dois sites de instituições públicas. Os resultados mostraram que o conteúdo dos serviços não estava acessível ao público analfabeto funcional. Em 2017, um trabalho (CAPRA; FERREIRA; TORRES. 2020) publicado num importante simpósio de Sistemas de Informação analisou o conteúdo textual de 26 serviços, de impacto social, de um importante órgão público federal. Os resultados mostraram que nenhum dos serviços estava acessível ao público analfabeto funcional, corroborando com resultados de outros artigos referenciados.

No Brasil, embora a acessibilidade de interfaces para analfabetos funcionais seja um tema abordado em congressos acadêmicos e periódicos da área da ciência da computação, ainda não é destaque em relação a outras limitações, como a visual e a auditiva. Uma pesquisa realizada em 2016 (GRANATTO; PALLARO; BIM, 2016) revelou que o analfabetismo funcional é o assunto com menos publicações nacionais.

\section{MÉTODO DE PESQUISA}

O presente trabalho, de caráter qualitativo e exploratório, baseou-se em um mapeamento sistemático, que orienta o pesquisador em como avaliar e interpretar os trabalhos relevantes para uma questão de pesquisa específica. Para isso, foi seguida a abordagem Systematic Literature Review (SLR) (KITCHENHAM, 2004; KITCHENHAM; CHARTERS, 2007). A abordagem SLR se baseia em diretrizes constituídas pelas seguintes fases que nortearam a presente pesquisa: 1) planejar, em que o pesquisador identifica a necessidade e elabora o protocolo que auxilie responder as questões que levaram ao mapeamento; 2) conduzir, em que o pesquisador identifica a base de pesquisa e seleciona os artigos para avaliá-los; e 3) relatar, em que os resultados são refinados e publicados em formato de relatório. 


\subsection{Planejamento do mapeamento sistemático}

Foram seguidas as seguintes etapas:

- Definição da principal questão da pesquisa: Quais aspectos podem influenciar no desenvolvimento de interfaces acessíveis para cuidadores analfabetos funcionais?

- Definição das questões específicas para responder à principal questão da pesquisa:

1. Qual país se destaca na realização de pesquisas sobre o analfabetismo funcional?

2. Quando as pesquisas sobre analfabetismo funcional começaram a ganhar destaque na comunidade de IHC?

3. Quais as principais bases acadêmicas destacam o tema?

4. Quais os principais domínios de sistema abordados nas pesquisas encontradas

5. Quais as principais contribuições para o público pesquisado?

Além dessas questões específicas, buscou-se identificar quais as principais convergências entre pesquisas nacionais e internacionais, com o intuito de refletir sobre possíveis lacunas nas pesquisas brasileiras.

- Definição das fontes para busca de trabalhos acadêmicos: decidiu-se por buscar artigos publicados entre os anos 2000 e 2019 e disponíveis nas bases acadêmicas com publicações em IHC mais conhecidas pelos autores: ACM Digital Library, IEEE, Scielo e Scopus.

- Definição dos critérios para seleção e exclusão dos artigos a serem lidos: foram selecionados os artigos completos, uma vez que estes possuíam conteúdo mais significativo para análise. De forma a conseguir extrair o maior número de artigos possível, as palavras no idioma português utilizadas como parâmetros de pesquisa foram: "acessibilidade", "web", "alfabetismo", "analfabetismo", "analfabetismo funcional", "analfabeto". Já no idioma inglês, utilizaram-se os termos: "accessibility", "literacy", "illiteracy", "functional illiteracy", "illiterate". Os artigos sem relação com o tema acessibilidade $w e b$, focado em analfabetismo funcional, foram excluídos (critério de exclusão). Evitou-se incluir, nos parâmetros, termos relacionados a "cuidadores" para que mais resultados pudessem ser extraídos.

\subsection{Condução do Mapeamento Sistemático}

Nesta fase do mapeamento, buscou-se selecionar os artigos e avaliá-los, extraindo dados relevantes para análise das informações. A Figura 2 apresenta o fluxo do mapeamento sistemático conduzido pelos autores, apresentando os resultados do total de artigos incluídos e excluídos em cada etapa.

Figura 2: Fluxo do Mapeamento Sistemático.

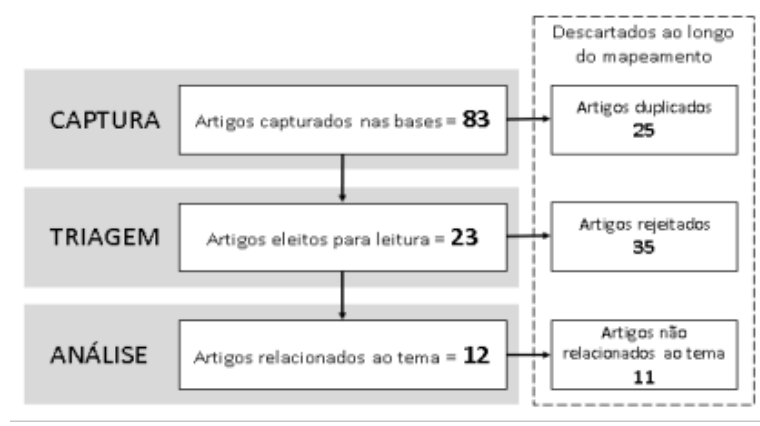

- Captura dos artigos: as publicações foram obtidas das bases citadas, de acordo com os parâmetros informados e exportados em arquivo . bib com informações sobre ano de publicação, autores, títulos e resumo. Foi gerado um arquivo para cada base. Para facilitar o planejamento e condução do mapeamento sistemático, os autores utilizaram uma ferramenta online gratuita Parsifal (PARSIFAL, 2018). Os arquivos .bib foram importados para a ferramenta e, no total, foram apresentados 83 artigos completos para análise, distribuídos da seguinte forma: ACM Digital Library = 16, IEEE $=21$, Scielo $=4$ e Scopus $=42$. A própria ferramenta Parsifal identificou 25 artigos duplicados. Desta forma, ficaram disponíveis para a fase seguinte 53 artigos.

- Triagem dos artigos: os títulos e resumos dos artigos importados para a ferramenta Parsifal foram lidos e analisados pelos autores. Aqueles que continham informações que faziam parte do escopo da pesquisa foram marcados na ferramenta como “aceitos" para a posterior leitura completa. Foram selecionados 23 artigos para leitura completa.

- Análise dos artigos selecionados: após a leitura e análise do conteúdo dos 23 artigos, as informações sobre cada trabalho foram armazenadas na ferramenta Parsifal. Após análise, foram elencados doze artigos como parte do escopo principal da pesquisa. 


\subsection{Relato dos Resultados}

Nesta fase, foi realizada a análise sobre a leitura completa dos artigos. Os resultados foram consolidados de forma a responder às questões específicas definidas na fase de planejamento, bem como a principal questão de pesquisa. Para entender essas questões e apresentar outras reflexões acerca do tema, foi elaborado mapa comparativo das publicações analisadas, que possibilitou identificar algumas convergências entre as pesquisas nacionais e internacionais.

\section{RESULTADOS E DISCUSSÃo}

Embora os resumos pudessem ser extraídos das análises após leitura completa dos artigos referenciados nesta pesquisa, decidiu-se pela inclusão de um extrato de cada artigo, conforme Tabela 1, a fim de facilitar a consulta e compreensão sobre as questões específicas que norteiam o mapeamento. Foram coletadas informações como: referência (nome/ano), país de realização da pesquisa e resumo. O resumo é um extrato de cada artigo após a leitura completa realizada pelos autores.

Foi elaborada uma matriz comparativa, apresentada na Figura 3 cuja finalidade foi facilitar a comparação das questões específicas da pesquisa.

\subsection{Análise Sobre as Questões Específicas do Ma- peamento}

A análise sobre todas essas questões está baseada na Tabela 1 e na Figura 3 Algumas perguntas iniciais foram elaboradas para conhecer como o analfabetismo funcional tem se destacado na comunidade de IHC, como as questões 1,2 e 3 . As questões 4 e 5 buscaram colaborar para responder à principal questão da pesquisa.

Em resposta à questão "1) Qual país se destaca na realização de pesquisas sobre o analfabetismo funcional?", a Índia e o Brasil são os que mais contêm publicações sobre acessibilidade para esse público, com quatro publicações cada país. No geral, o continente asiático (Índia e Paquistão) se destaca com metade das publicações sobre o tema (seis artigos). Já no continente americano, o Brasil se destaca como país preocupado com o acesso à web por analfabetos funcionais, totalizando quatro publicações. Esperava-se que países em processo de crescimento apresentassem mais pesquisas em relação ao tema, especialmente o Brasil, uma vez que se estima que $14,1 \%$ dos brasileiros acima de 15 anos sejam analfabetos funcionais (INEP, 2020) ou $29 \%$, segundo o Indicador de Alfabetismo Funcional (INAF, 2018).
Em resposta à pergunta “2) Quando as pesquisas sobre analfabetismo funcional começaram a ganhar destaque na comunidade de IHC?", percebe-se que o assunto começou a se destacar a partir de 2007. Entretanto, mesmo tendo a média de uma publicação anual, pela matriz comparativa é possível perceber que trabalhos relacionados a esse tema não são constantes, havendo intervalos de anos em que não foram encontradas publicações, como 2008, 2010 e 2016. Cabe ressaltar que, de acordo com o intervalo de anos pesquisados (de 2000 a 2018), somente em 2007 foi identificada a primeira publicação sobre o tema.

Quanto à questão “3) Quais as principais bases acadêmicas destacam o tema?", o mapeamento mostrou que a Scopus possui metade das publicações (seis artigos), acompanhada da ACM Digital Library com cinco artigos. Já a IEEE possui somente uma publicação. Além disso, ao analisar a matriz comparativa, a ACM Digital Library foi a base que disponibilizou as primeiras publicações sobre acessibilidade $w e b$ para analfabetos funcionais. De 2012 a 2017, a Scopus foi a base predominante nas publicações sobre o tema, principalmente acerca de trabalhos relacionados aos países asiáticos, como Índia e Paquistão. Pode-se atribuir o destaque dessas bases ao fato de possibilitarem também publicações de trabalhos apresentados em congressos e simpósios internacionais, o que ajuda na divulgação das pesquisas para a comunidade.

Sobre a questão “4) Quais os principais domínios de sistema abordados nas pesquisas encontradas?", a interface de sites foi a mais pesquisada, com seis publicações. A matriz comparativa também mostra que as interfaces de sites foram os domínios predominantemente pesquisados de 2007 a 2012 pela Índia e Brasil. Depois, o domínio de pesquisa ficou mais diversificado, com pesquisas sobre sistemas e aplicativos em diferentes países. Sobre o conteúdo textual, cuja simplicidade de escrita é um dos requisitos básicos que contribui para melhor entendimento do analfabeto funcional (MARTINS et al., 2003; HUENERFAUTH, 2002, BARBOZA; NUNES 2007), somente um pesquisador brasileiro publicou sobre o tema, propondo o desenvolvimento de uma tecnologia assistiva para facilitar a leitura do conteúdo textual em português (WATANABE et al. 2009).

Em resposta à questão "5) Quais as principais contribuições para o público pesquisado?”, destacam-se os aplicativos e recomendações que, somados, apresentaram $67 \%$ de relevância sobre as contribuições de modelo e sistema (33\%). Já os aplicativos se destacaram por estarem relacionados aos equipamentos mobiles (DWIVEDI et al., 2013; MESFIN et al., 2015) e 
Tabela 1: Resumo dos Artigos Identificados no Mapeamento Sistemático

\begin{tabular}{|c|c|c|c|c|}
\hline \multicolumn{3}{|c|}{ Referência } & País & Resumo \\
\hline \multicolumn{2}{|c|}{ Medhi, Prasad e Toyama } & $(2007)$ & Índia & $\begin{array}{l}\text { O artigo discutiu diferentes tipos de representações audiovisuais para ilustrar conceitos aos } \\
\text { usuários analfabetos e semianalfabetos. Para isso, os autores realizaram uma pesquisa com } \\
\text { duzentos usuários utilizando treze sintomas diferentes de saúde. Os resultados mostraram } \\
\text { que: a descrição por voz geralmente ajuda na velocidade da compreensão, mas a informação } \\
\text { audiovisual pode ser confusa para analfabetos; desenhos à mão que podem se sair melhores } \\
\text { do que fotografias; e o valor relativo de imagens dinâmicas e imagens estáticas é misto. }\end{array}$ \\
\hline \multicolumn{2}{|c|}{ Watanabe et al. (2009) } & & Brasil & $\begin{array}{l}\text { O artigo discutiu questões sobre o desenho de interação para analfabetos funcionais e } \\
\text { apresentou o aplicativo Facilita, uma tecnologia assistiva elaborada para ajudar os } \\
\text { usuários com baixa escolaridade a entender o conteúdo textual das páginas de internet. } \\
\text { O aplicativo foi baseado em técnicas de sumarização e simplificação textual. Por } \\
\text { tratar-se de um protótipo, não foram realizadas interações com usuários reais. }\end{array}$ \\
\hline Watanabe 2 & 2010) & & Brasil & $\begin{array}{l}\text { O artigo teve como objetivo complementar o estudo de WATANABE, } 2009 \text {, com } \\
\text { reflexões importantes sobre a existência de diretrizes de acessibilidade que, infelizmente, } \\
\text { não garantem garantir que desenvolvedores web estejam preparados para } \\
\text { elaborar o conteúdo de um site acessível. }\end{array}$ \\
\hline \multicolumn{2}{|c|}{ Hamouda et al. (2011) } & & Egito & $\begin{array}{l}\text { O artigo apresentou o Face-Back, um serviço de comunicação bidirecional que visou unir } \\
\text { dois tipos de pessoas: analfabetas e alfabetizadas. Os autores elaboraram um diagrama de } \\
\text { afinidade para identificar as áreas temáticas comuns aos dois grupos. O sistema resultou } \\
\text { em bons resultados e como trabalho futuro foi desenvolvê-lo para iPhone. }\end{array}$ \\
\hline Capra et al. 2 & $2012 b$ & & Brasil & $\begin{array}{l}\text { O artigo pesquisou o comportamento de analfabetos funcionais na web por meio de } \\
\text { uma investigação com pesquisadores (estudo etnográfico), identificando características } \\
\text { importantes que deviam ser consideradas em uma avaliação de acessibilidade com esse } \\
\text { público. Por fim, foi realizado um estudo de caso, a fim de verificar como realizar avaliações } \\
\text { de acessibilidade web com a participação de analfabetos funcionais. }\end{array}$ \\
\hline \multicolumn{3}{|c|}{ Mahmood, Shahzadi e Tariq (2014) } & Paquistão & $\begin{array}{l}\text { O artigo discorreu sobre uma pesquisa cujo objetivo foi propor uma solução eficaz e eficiente } \\
\text { para atender usuários não letrados dentro de um sistema gerenciador de conteúdo. Foram } \\
\text { conduzidos testes com usuários, apresentando interfaces com gravação de voz, vídeos e } \\
\text { animações. O autor apresentou um vasto referencial teórico com trabalhos relacionados } \\
\text { às interfaces livres de textos e com uso de áudio para usuários analfabetos e semianalfabetos. }\end{array}$ \\
\hline \multicolumn{2}{|c|}{ Das, Mikko e Gururaj } & (2015) & Índia & $\begin{array}{l}\text { O artigo propôs um modelo baseado de negócio que pudesse fornecer serviços a uma } \\
\text { população pobre da Índia e, frequentemente, analfabeta. Os autores sugeriram um modelo } \\
\text { geral de negócios para diferentes tipos de serviços e um fluxo de dinheiro entre os } \\
\text { participantes, a fim de explorar a possibilidade de aplicações comerciais usando a web } \\
\text { falada direcionada ao público da base da pirâmide. }\end{array}$ \\
\hline \multicolumn{3}{|c|}{ Mesfin et al. (2015) } & Etiópia & $\begin{array}{l}\text { O artigo teve como objetivo sugerir uma solução para uma aplicação de dinheiro digital } \\
\text { especificamente para analfabetos das localidades rurais da Etiópia. Os pesquisadores } \\
\text { identificaram que uma abordagem alternativa foi o uso de interfaces naturais, utilizando-se } \\
\text { de fotos de notas de dinheiro. Os autores relataram que esses usuários se sentiam } \\
\text { confortáveis com interfaces não textuais. }\end{array}$ \\
\hline \multicolumn{3}{|c|}{ Khan et al. (2017) } & Paquistão & $\begin{array}{l}\text { O artigo apresentou uma pesquisa exploratória conduzida para projetar uma solução de } \\
\text { tecnologia de informação e comunicação (TIC) a fim de ajudar a população analfabeta de } \\
\text { uma localidade do Paquistão a procurar um emprego apropriado, de forma que o usuário } \\
\text { não precisasse de assistência humana para usá-lo. Os autores propuseram um site } \\
\text { desenvolvido em conteúdo gráfico, seguindo o princípio "simples é melhor"e baseando-se } \\
\text { em imagens que representavam trabalhos domésticos, jardinagem, segurança, barbeiro, } \\
\text { entre outros. O tempo de conclusão das tarefas foi melhor em comparação com outras } \\
\text { soluções. }\end{array}$ \\
\hline
\end{tabular}


Figura 3: Matriz Comparativa das Publicações.

\begin{tabular}{|c|c|c|c|c|c|c|c|c|c|c|c|}
\hline PAIS I ANO & 2007 & 2008 & 2009 & 2010 & 2011 & 2012 & 2013 & 2014 & 2015 & 2016 & 2017 \\
\hline India & 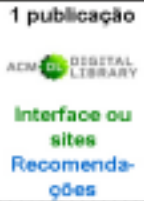 & \multirow{5}{*}{ 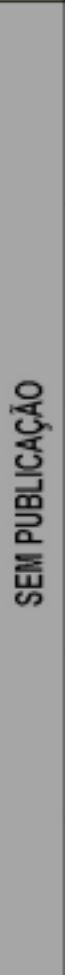 } & HABS & \multirow{5}{*}{ 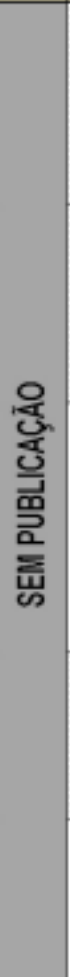 } & & $\begin{array}{l}1 \text { publicação } \\
\text { ScOpuS" } \\
\text { Interface } \\
\text { sites } \\
\text { Modelo }\end{array}$ & $\begin{array}{l}1 \text { publioagao } \\
\text { ScOpuS } \\
\text { Aplicativo } \\
\text { Aplicativo }\end{array}$ & & $\begin{array}{c}1 \text { publioagao } \\
\text { ScOpuS } \\
\text { Sistema } \\
\text { Modelo }\end{array}$ & \multirow{5}{*}{ 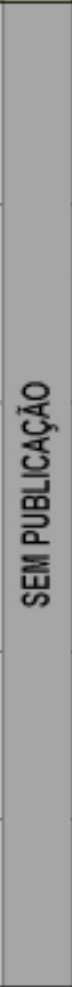 } & \\
\hline Paquistao & & & a & & & & & $\begin{array}{l}1 \text { publicação } \\
\text { ScOpuS } \\
\text { Sistema } \\
\text { Sistema }\end{array}$ & & & $\begin{array}{c}1 \text { publicagao } \\
\text { ScOpuS } \\
\text { Interface e } \\
\text { sites } \\
\text { Reoomenda: } \\
\text { çóes }\end{array}$ \\
\hline Brasil & & & 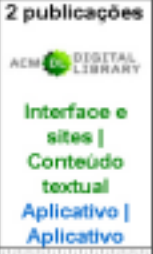 & & 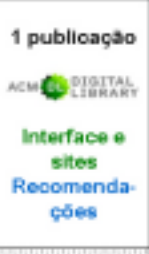 & $\begin{array}{c}1 \text { publioagao } \\
\text { ScOpuS" } \\
\begin{array}{c}\text { Interface e } \\
\text { sites }\end{array} \\
\text { Recomenda. } \\
\text { çồ }\end{array}$ & & & & & \\
\hline Ettopia & & & & & & & & & $\begin{array}{l}1 \text { publicacaso } \\
\text { IEEE Xplore' } \\
\text { Aplicativo } \\
\text { Aplicativo }\end{array}$ & & \\
\hline Egito & & & & & 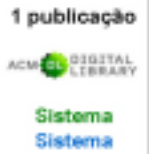 & & & & & & \\
\hline
\end{tabular}

às tecnologias assistivas (WATANABE et al., 2009). A matriz comparativa revelou a Índia como o país com a melhor distribuição de publicações com objeto de estudo e contribuições variadas. O Brasil aparece como o país referência em publicações sobre o tema de 2009 a 2012, com contribuições centradas em aplicativos e recomendações. Especialmente sobre as recomendações para a área de saúde, o trabalho de Medhi, Prasad e Toyama (2007) mostrou que o uso de recursos audiovisuais pode deixar os usuários analfabetos funcionais confusos para entenderem algum contexto relacionado a sintomas de saúde.

Ainda analisando as contribuições, é possível verificar que os propósitos das recomendações são distintos. Na Índia e Paquistão, estas estão atreladas ao desenvolvimento de interface. Observou-se que algumas contribuições do Brasil e outros países são recomendações e aplicativos. Outra convergência importante são pesquisas cujos resultados são aplicativos para o público analfabeto funcional, especialmente no que se refere a tornar a agricultura mais produtiva, como facilitar o uso do dinheiro digital por esse público. No entanto, o Brasil contribuiu com aplicativos mais voltados a tecnologias assistivas, com vistas a transformar uma página web compreensível ao analfabeto funcional.

\subsection{Análise Sobre a Questão Principal do Mapea- mento Sistemático}

A partir da análise sobre os doze trabalhos identificados, foi possível refletir sobre cada contribuição, a fim de responder à principal questão do mapeamento: Quais aspectos podem influenciar no desenvolvimento de interfaces acessíveis para cuidadores analfabetos funcionais?

Além do mapeamento evidenciar que a pesquisa sobre o tema analfabetismo é mais presente em países subdesenvolvidos, especialmente no campo da saúde, também corroborou com a pesquisa de Granatto (GRANATTO; PALLARO; BIM, 2016), segundo a qual a acessibilidade $w e b$ para analfabetos funcionais é o tema com menos publicações no Simpósio Brasileiro sobre Fatores Humanos em Sistemas Computacionais (SBSC), tendo destaque no evento até 2011, com tímidos $13 \%$ das publicações.

Acerca dos principais sistemas estudados e contribuições, foram mapeados os seguintes aspectos que podem ser considerados no desenvolvimento de uma in- 
terface como facilitadores para garantir interfaces acessíveis a cuidadores analfabetos funcionais:

- Dar preferência a interfaces com uso de imagens e menos textos (DWIVEDI et al., 2013, MESFIN et al. 2015);

- Evitar os recursos audiovisuais (MEDHI; PRASAD; TOYAMA, 2007);

- Para informações que precisam ser rapidamente compreendidas, pode-se utilizar recursos de descrição por voz (MEDHI; PRASAD; TOYAMA 2007; DAS; MIKKO; GURURAJ, 2015);

- Dependendo do que será representado, o uso de recursos de ilustrações, como desenhos à mão (KHAN et al., 2017) ou ícones (MAITI; SAMANTA, 2012), podem ser mais bem compreendidos do que fotografias (MEDHI; PRASAD; TOYAMA, 2007);

- O uso de interfaces naturais, como as fotografias, pode ser utilizado para os casos onde as ilustrações não consigam representar a informação que se deseja transmitir (MESFIN et al., 2015);

- Para sistemas gerenciadores de conteúdo, o uso de recursos de voz pode auxiliar na localização da informação (MAHMOOD; SHAHZADI; TARIQ 2014).

De acordo com os aspectos mapeados, o mapeamento possibilitou conhecer aspectos importantes a serem considerados no desenvolvimento de tecnologia acessível para cuidadores analfabetos funcionais, ainda que alguns artigos tivessem foco em outras áreas que não fossem da saúde.

\section{CONSIDERAÇÕES FINAIS}

A evolução das tecnologias de comunicação, em especial a internet, trouxe novas perspectivas de comunicação entre as pessoas. Mas também trouxe muitos desafios, como promover o acesso à internet às pessoas com dificuldades nas habilidades de leitura e escrita, ou seja, analfabetas funcionais.

No Brasil, são consideradas analfabetas funcionais pessoas com escolaridade com menos de quatro anos completos de estudo (IBGE, 2010, INEP, 2020, RIBEIRO; VÓVIO; MOURA, 2002). Neste contexto, os cuidadores de idosos são formados, em sua maior parte, por pessoas com baixa escolaridade (JESUS; ORLANDI; ZAZZETTA, 2018). Desta forma, o analfabetismo funcional emerge como um tópico social importante a ser considerado no design de uma interface, uma vez que boa parcela deste público, além de acessar a internet por meio de diferentes dispositivos, tem significativa representatividade nas tarefas de cuidado do idoso. Assim, este artigo teve como objetivo analisar as contribuições disponíveis na literatura acerca da acessibilidade web para analfabetos funcionais, a fim de identificar os aspectos que podem tornar a web acessível aos cuidadores analfabetos funcionais e possíveis lacunas para pesquisas futuras.

Para isso, foi realizado um mapeamento sistemático em 58 artigos completos publicados entre 2000 e 2019 em quatro bases acadêmicas internacionais. Foram identificadas doze pesquisas relacionadas ao tema, que possibilitaram responder à principal questão da pesquisa: Quais aspectos podem influenciar no desenvolvimento de interfaces acessíveis para cuidadores analfabetos funcionais?

Como uma importante reflexão, os autores deste artigo alertam que, mesmo o analfabetismo funcional sendo tema de algumas pesquisas apresentadas em eventos na comunidade de IHC, pesquisar sobre acessibilidade $w e b$ para esse público não tem sido tratado como tema relevante nos últimos anos, dado o número restrito (doze) de publicações identificadas em quatro grandes bases acadêmicas. Essa reflexão corrobora com a análise realizada por Granatto, Pallaro e Bim (2016), segundo a qual, até 2011 , somente $13 \%$ das publicações em IHC abordavam o tema.

O mapeamento realizado para este trabalho possibilitou identificar importantes abordagens nos artigos analisados, especialmente relacionadas ao cunho socioeconômico, à agricultura e até ao processo de avaliação $w e b$ com a participação de analfabetos funcionais.

Embora sendo um tema pouco representativo na área da saúde, com somente um trabalho identificado (MEDHI; PRASAD; TOYAMA, 2007), todos os trabalhos analisados possibilitaram identificar fatores interessantes que podem influenciar no desenvolvimento de interfaces acessíveis a cuidadores analfabetos funcionais, como uso de imagens, desenhos, recursos audiovisuais, fotografias e notação por voz.

Os resultados da presente pesquisa podem ser considerados como uma oportunidade para contribuir para uma reflexão de pesquisadores (especialmente brasileiros) sobre a importância do tema, não só para a comunidade de IHC, mas também de outras áreas relacionadas a tecnologias voltadas aos cuidadores. É importante destacar que a expectativa de vida tem crescido a cada década, o que evidencia a necessidade de contar com a colaboração desses parceiros, independente de serem remunerados ou não. 
ACESSIBILIDADE DE INTERFACES WEB PARA CUIDADORES COM BAIXA ESCOLARIDADE: ASPECTOS E REFLEXÕES

\section{REFERÊNCIAS}

ALMEIDA, K. M. V. d. Avaliação do alfabetismo funcional em saúde em cuidadores de idosos. Dissertação (mathesis) - Universidade Estadual Paulista Júlio de Mesquita Filho, 2017. 62 f.

BARBOZA, E. M. F.; NUNES, E. M. d. A. A inteligibilidade dos websites governamentais brasileiros e o acesso para usuários com baixo nível de escolaridade. Revista Ciência da Informação, Ibict, v. 2, n. 2, p. 19-33, 2007.

BENJAMIN, K.; FERREIRA, S. B. L.; CAPPELLI, C.; CAPRA, E. P.; SILVEIRA, D. S. D. Sites governamentais: Acessíveis para analfabetos funcionais? In: Encontro de Administração da Informação. Bento Gonçalves - RS: IV EnAdI, 2013.

CAPRA, E. P.; FERREIRA, S. B. L.; SILVEIRA, D. S. D.; RIBEIRO, B. B.; MODESTO, D. M. Evaluation of web accessibility from the perspective of functional illiteracy. In: Proceedings of the 10th Brazilian Symposium on Human Factors in Computing Systems and the 5th Latin American Conference on Human-Computer Interaction. Pernambuco: IHC, 2011. p. 280-288.

CAPRA, E. P.; FERREIRA, S. B. L.; SILVEIRA, D. S D.; FERREIRA, A. O. Evaluation of web accessibility: An approach related to functional illiteracy. Procedia Computer Science, Elsevier, v. 14, p. 36-46, 2012.

CAPRA, E. P.; FERREIRA, S. B. L.; SILVEIRA, D. S. D.; ALVES, A. D. S. Protocolos para avaliação da acessibilidade web com a participação de analfabetos funcionais. In: PARALLEL SESSION AND PUBLICATION. International Conference on Information Systems and Technology Management. São Paulo: 9th CONTECSI, 2012.

CAPRA, E. P.; FERREIRA, S. B. L.; TORRES, B. A. Verificação da adequação do conteúdo textual dos serviços web públicos brasileiros para usuários com baixo letramento. Brazilian Journal of Development, v. 6 , n. 12 , p. 97386-97402, 2020.

CARDOSO, R. d. S. S.; SÁ, S. P. C.; DOMINGOS, A. M.; SABÓIA, V. M.; MAIA, T. N.; PADILHA, J. M. F. d. O.; NOGUEIRA, G. d. A. Tecnologia educacional: um instrumento dinamizador do cuidado com idosos. Revista Brasileira de Enfermagem, SciELO Brasil, v. 71, n. 1, p. 786-792, 2018.

CASTELL, S.; LUKE, A.; EGAN, K. Literacy, society, and schooling: A reader. 1. ed. Cambridge: Cambridge University Press, 1986.
CETIC-C6. Indicador C6 - Usuários de Internet, por Atividades Realizadas na Internet -

Busca de Informação. 2019. Disponível em: $<$ https://cetic.br/tics/domicilios/2019/individuos/C6/>

COLOMÉ, I. C. d. S.; MARQUI, A. B. T. de; JAHN, A. do C.; RESTA, D. G.; CARLI, R. D.; WINCK, M. T.; NORA, T. T. D. Cuidar de idosos institucionalizados: características e dificuldades dos cuidadores. Revista Eletrônica de enfermagem, v. 13, n. 2, p. 306-12, 2011.

DAS, H.; MIKKO, R.; GURURAJ, M. Ecosystem modeling for mobile voice based services; rural india's bottom of the pyramid view. In: International Conferences e-Health, e-Commerce and Digital Marketing. Portugal: Eamsa, 2015.

DIEL, B. L. Analfabetismo funcional: a inserção do cidadão analfabeto no mercado de trabalho. Eventos Pedagógicos, v. 5, n. 2, p. 373-380, 2014.

DWIVEDI, S.; PARSHAV, V.; SHARMA, N.; KUMAR, P.; CHHABRA, S.; GOUDAR, R. Using technology to make farming easier and better: Simplified e-farming support (sefs). In: IEEE. 2013 International Conference on Human Computer Interactions (ICHCI). Chennai, 2013. p. 1-6.

ELLCESSOR, E. Blurred lines: Accessibility, disability, and definitional limitations. First Monday, v. 20 , n. 9,2015

FERREIRA, S. B. L.; NUNES, R. e-Usabilidade. 1. ed. Rio de Janeiro: LTC, 2008.

FURTADO, G. F. F. F.; SILVA, A. C. L. S. L.; AMORIM, L. L. A. A. B.; SOUSA, T. M. D. S. M.; QUEIROZ, G. A. A. Q. A.; SILVA, E. I. D. S. S.; PAUL, I. C. P. D. N.; NASCIMENTO, P.; OTHERS. Perfil do idoso da cidade de caridade-ce. Conexões-ciência e tecnologia, v. 14, n. 4, p. 07-21, 2020 .

GRANATTO, C. F.; PALLARO, M. A.; BIM, S. A. Acessibilidade digital: Revisão sistemática dos artigos do simpósio brasileiro sobre fatores humanos em sistemas computacionais. In: Simpósio Brasileiro sobre Fatores Humanos em Sistemas Computacionais. São Paulo: IHC, 2016. v. 15.

HAMOUDA, H. A.; HUSSEIN, M. M.; SHARAFEL-DEEN, M. H.; ABDEL-AZIZ, N. M.; HANNA, S. M. Face-back: who is the illiterate again? In: CHI'11 Extended Abstracts on Human Factors in 
ACESSIBILIDADE DE INTERFACES WEB PARA CUIDADORES COM BAIXA ESCOLARIDADE: ASPECTOS E REFLEXÕES

Computing Systems. 11. ed. Vancouver, Canadá: CHI $\quad$ MAHMOOD, Z.; SHAHZADI, S. S.; TARIQ, S.

EA'11, 2011. p. 953-958.

Content management and user interface for uneducated

HUENERFAUTH, M. P. Developing design recommendations for computer interfaces accessible to illiterate users. Dissertação (mathesis) — University College Dublin, Dublin, 2002. 136 f.

IBGE. Séries históricas e Estatísticas. Tema e subtemas: educação, alfabetização e instrução - taxa de analfabetismo funcional (definição e comentário) - período de 2001-2009. Brasil, 2010. Disponível em: <https://seriesestatisticas.ibge.gov. br/series.asp 2 no $=4 \& o p=0 \& v$ codigo $=$ PD 384\&t $=$ taxa-analfabetismo-funcional>.

INAF. Indicador de Alfabetismo Funcional. Brasil, 2018. Http://acaoeducativa.org.br/wpcontent/uploads/2018/08/Inaf2018Relat \%C3\%B3rioResultados-Preliminares_v08Ago2018.pdf.

INEP. Relatório do $3^{\circ}$ ciclo de monitoramento das metas do Plano Nacional de Educação - 2020. Brasil: [s.n.], 2020. Instituto Nacional de Estudos e Pesquisas Educacionais Anísio Teixeira. Disponível em: <http: //portal.inep.gov.br/informacao-da-publicacao/-/asset_ publisher/6JYIsGMAMkW1/document/id/6975827>

JESUS, I. T. M. d.; ORLANDI, A. A. d. S.; ZAZZETTA, M. S. Sobrecarga, perfil e cuidado: cuidadores de idosos em vulnerabilidade social. Revista Brasileira de Geriatria e Gerontologia, SciELO Brasil, v. 21, n. 2, p. 194-204, 2018.

KHAN, I. A.; HUSSAIN, S. S.; SHAH, S. Z. A.; IQBAL, T.; SHAFI, M. Job search website for illiterate users of pakistan. Telematics and Informatics, Elsevier, v. 34, n. 2, p. 481-489, maio 2017.

KITCHENHAM, B. Procedures for performing systematic reviews. Keele, UK, Keele University, v. 33, n. 2004, p. 1-26, 2004.

KITCHENHAM, B.; CHARTERS, S. Guidelines for performing systematic literature reviews in software engineering. University of Durham, 2007.

KODAGODA, N.; WONG, B. W. Effects of low \& high literacy on user performance in information search and retrieval. In: Proceedings of the 22nd British HCI Group Annual Conference on People and Computers: Culture, Creativity, Interaction BCS-HCI08. Liverpool: United Kingdom, 2008. v. 1, p. 173-181. people. In: MARCUS, A. (Ed.). Design, User Experience, and Usability. User Experience Design for Everyday Life Applications and Services. Cham: Springer International Publishing, 2014. p. 432-441. ISBN 978-3-319-07635-5.

MAITI, S.; SAMANTA, D. Icon-based representation of web information. In: IEEE. 2012 4th International Conference on Intelligent Human Computer Interaction (IHCI). Kharagpur, Índia: IHCI, 2012. p. 1-6.

MARTINS, I.; CARVALHO, L.; FERREIRA, L.; CALHAÚ, M.; BENÍCIO, M. Aspectos da interação humano-computador em sistemas para jovens e adultos não alfabetizados. In: CLIHC. Proceedings of the 2003 Latin American Conference on Human-Computer Interaction. Rio de Janeiro, 2003. p. 235-238.

MEDHI, I.; PRASAD, A.; TOYAMA, K. Optimal audio-visual representations for illiterate users of computers. In: Proceedings of the 16th international conference on World Wide Web. Alberta, Canadá: IW3C2, 2007. p. 873-882.

MESFIN, W.; GRØNLI, T.-M.; GHINEA, G.; YOUNAS, M. A mobile money solution for illiterate users. In: IEEE. 2015 IEEE International Conference on Mobile Services. Coimbra, Portugal: IEEE MS, 2015. p. 328-335.

NIELSEN, J. Usabilidade na web. 1. ed. Rio de Janeiro: Elsevier Brasil, 2007.

PARSIFAL. Parsifal LTDA. 2018. Disponível em: $<$ https://parsif.al/>

PNAD. Pesquisa Nacional de Amostra por Domicílios - Educação 2017. 2017. Disponível em: <https://www.ibge.gov.br/estatisticas/sociais/trabalho/ 17270-pnad-continua.html?edicao $=21073 \& \mathrm{t}=$ sobre $>$

PRAKASH, K. B.; ANANTHAN, T.; RAJAVARMAN, $\mathrm{V}$. Neural network framework for multilingual web documents. In: IEEE. 2014 International Conference on Contemporary Computing and Informatics (IC3I). Mysore, Índia: IC3I, 2014. p. 392-397.

RIBEIRO, V. M. Alfabetismo funcional: referências conceituais e metodológicas para a pesquisa. Educação \& Sociedade, SciELO Brasil, v. 18, n. 1, p. 144-158, 1997. 
RIBEIRO, V. M.; VÓVIO, C. L.; MOURA, M. P. Letramento no brasil: alguns resultados do indicador nacional de alfabetismo funcional. Educação \& Sociedade, SciELO Brasil, v. 23, n. 81, p. 49-70, 2002 .

SACRAMENTO, C.; FERREIRA, S. B. L.; CAPRA, E. P.; GARCIA, A. C. B. Accessibility and communicability on facebook: A case study with brazilian elderly. First Monday, v. 24, n. 1, 2019.

SILVA, E.; TORRES, B.; SACRAMENTO, C.; CAPRA, E. P.; FERREIRA, S. B. L.; GARCIA, A. C. B. Privacy: What is the research scenario in brazilian symposium ihc? In: Proceedings of the 17th Brazilian Symposium on Human Factors in Computing Systems. Belém - PA: IHC, 2018. p. 1-8.

VÁGVÖLGYI, R.; COLDEA, A.; DRESLER, T.; SCHRADER, J.; NUERK, H.-C. A review about functional illiteracy: Definition, cognitive, linguistic, and numerical aspects. Frontiers in psychology, Frontiers, v. 7, n. 1, p. 1617, 2016.

WAI. Web Accessibility Initiative. 2020. Disponível em: <https://www.w3.org/WAI/>

WATANABE, W. M. Facilita: reading assistance to the functionally illiterate. In: Proceedings of the 2010 International Cross Disciplinary Conference on Web Accessibility (W4A). Raleigh - NC, USA: W4A, 2010. p. 1-2.

WATANABE, W. M.; JUNIOR, A. C.; UZÊDA, V. R.; FORTES, R. P. d. M.; PARDO, T. A. S.; ALUÍSIO, S. M. Facilita: reading assistance for low-literacy readers. In: Proceedings of the 27th ACM international conference on Design of communication. Indiana, USA: SIGDOC, 2009. p. 29-36.

WCAG. World Wide Web Consortium. 2018. Versão 2.1. 20 de Setembro de 2018. Disponível em: <https://www.w3.org/TR/WCAG21/> 